\title{
3 Research Square \\ Outcomes of COVID-19 and risk factors in patients with cancer - a cohort study
}

\section{Manju Sengar}

Tata Memorial Centre, Homi Bhabha National Institute

Girish Chinnaswamy

Tata Memorial Centre, Homi Bhabha National Institute

\section{Priya Ranganathan}

Tata Memorial Centre, Homi Bhabha National Institute

\section{Apurva Ashok}

Tata Memorial Centre, Homi Bhabha National Institute

\section{Shilpushp Bhosale}

Tata Memorial Centre, Homi Bhabha National Institute

\section{Sanjay Biswas}

Tata Memorial Centre, Homi Bhabha National Institute

\section{Pankaj Chaturvedi}

Tata Memorial Hospital

\section{Chetan Dhamne}

Tata Memorial Centre, Homi Bhabha National Institute Jigeeshu Divatia

Tata Memorial Centre, Homi Bhabha National Institute

\section{Karishma Dsa}

Tata Memorial Centre, Homi Bhabha National Institute

\section{Hasmukh Jain}

Tata Memorial Centre, Homi Bhabha National Institute

\section{Sarbani Laskar}

Tata Memorial Centre, Homi Bhabha National Institute

Nirmalya Moulik

Tata Memorial Centre, Homi Bhabha National Institute

Naveen Mummudi

Tata Memorial Centre, Homi Bhabha National Institute

\section{Sindhu Nair}

Tata Memorial Centre, Homi Bhabha National Institute

\section{Lingaraj Nayak}

Tata Memorial Centre, Homi Bhabha National Institute 
Tata Memorial Centre, Homi Bhabha National Institute

\section{Shraddha Patkar}

Tata Memorial Centre, Homi Bhabha National Institute

\section{Preeti Pawaskar}

Tata Memorial Centre, Homi Bhabha National Institute

\section{Anant Ramaswamy}

Tata Memorial Centre, Homi Bhabha National Institute

\section{Omshree Shetty}

Tata Memorial Centre, Homi Bhabha National Institute

\section{Arjun Singh}

Tata Memorial Centre, Homi Bhabha National Institute

\section{Sridhar Epari}

Tata Memorial Hospital

\section{Jayashree Thorat}

Tata Memorial Centre, Homi Bhabha National Institute

\section{Rajendra Badwe}

Tata Memorial Centre, Homi Bhabha National Institute

C S Pramesh ( $\square$ cspramesh@gmail.com )

Tata Memorial Centre, Homi Bhabha National Institute https://orcid.org/0000-0002-3635-0083

\section{Article}

Keywords: COVID-19, SARS CoV2, cancer, risk factors

Posted Date: November 8th, 2021

DOI: https://doi.org/10.21203/rs.3.rs-1002083/v1

License: (c) (i) This work is licensed under a Creative Commons Attribution 4.0 International License.

Read Full License

Version of Record: A version of this preprint was published at Nature Cancer on April 4th, 2022. See the published version at https://doi.org/10.1038/s43018-022-00363-4. 


\section{Abstract \\ Background}

Patients with cancer who develop COVID-19 are at higher risk for severe disease and death. We aimed to study the outcomes of patients with cancer who were infected with SARS CoV2, and identify risk factors for adverse outcomes after COVID-19.

\section{Methods}

We included patients with cancer, who were diagnosed with SARS CoV2 between 11th April 2020 and February 2021 at a tertiary referral cancer centre in India. We collected data on age, sex, coexisting medical conditions, type of cancer, intent of cancer management, cancer treatment details, and severity and outcome of COVID-19. The primary outcome was a composite of severe COVID (defined as grade 6 or more on the WHO ordinal scale) or death within 30 days. We performed multivariable logistic regression analysis to identify risk factors for severe COVID or death.

\section{Results}

We obtained data on 1253 patients with cancer and SARS CoV2 infection, of whom 293 (23.6\%) had hematological malignancies. The severity of COVID was mild (grade 1 to 3 on WHO ordinal scale) in 1014 $(81 \%)$ patients, moderate (WHO grade 4 or 5) in $167(13 \%)$ patients and severe (WHO grade 6 or 7 ) in 72 (6\%) patients. The primary outcome was seen in 160 patients $(12.8 \%)$ and the all-cause 30 -day mortality was $10.9 \%$ (138 deaths). Advanced age [adjusted OR $1.84(0.86 ; 3.94)$ ], history of smoking [aOR 1.78 $(1.10 ; 2.91)]$, palliative intent of treatment [aOR 3.57 (2.48 to 5.12)] and presence of more than 2 comorbidities [aOR 1.66 (1.03 to 2.67)] were significant risk factors for severe COVID or death. Advanced age and palliative intent of treatment remained significant risk factors for 30-day mortality. Recent systemic anti-cancer therapy, sex or cancer type did not influence outcomes.

\section{Conclusion}

Most patients with cancer who developed COVID-19 in our setting had mild disease; the elderly and those treated with palliative intent were at higher risk of severe COVID-19 or death. Recent cancer therapy did not impact COVID-19 severity or outcomes suggesting that in most patients with cancer, the management of cancer should continue uninterrupted during the pandemic.

\section{Background And Introduction}

The current COVID-19 pandemic has infected more than 239 million people globally, causing nearly 5 million deaths. ${ }^{1}$ Global data suggest that increasing age, presence of concurrent illnesses and 
immunosuppression are some of the risk factors associated with poor outcomes after COVID-19. ${ }^{2,3}$ Patients with cancer are often immunosuppressed by the disease, as well as its treatment, and are at higher risk; in addition, some of the important predisposing factors for cancer like smoking and obesity are also contributory factors for adverse outcomes after COVID-19. Overall, patients with cancer have been found to have higher rates of severe disease and fatality after COVID-19 than the general population. ${ }^{4}$ The presence of additional comorbidities, poor performance status, specific cancer types and recent systemic anti-cancer therapy have been variably identified as adverse prognostic factors in patients with cancer and COVID-19; ${ }^{4-7}$ however, the actual risk associated with some of these factors remains unclear.

India has seen 34 million individuals infected with COVID-19, resulting in 450,000 deaths. ${ }^{1}$ Case fatality rates in general have been lower in India compared to other countries, especially Western Europe and the United States. ${ }^{1}$ While this has been attributed to under-reporting of cases (and deaths), the difference cannot be explained on this basis alone. ${ }^{8}$ It is likely that there are other factors, possibly acquired immunity due to the population being infected by other coronaviruses in the past, and other less known factors, which may contribute to the low fatality rate. ${ }^{9}$ There is very little data from India on the outcomes of COVID-19 in patients with cancer. ${ }^{10-12}$ Given that cancer treatment should be considered a priority, reliable data on outcomes of COVID-19 in patients with cancer are necessary to guide management during future surges of the pandemic. The aim of this study was to assess the short-term outcomes of COVID-19 in patients with cancer at a tertiary-referral cancer centre in Western India, and to identify risk factors for adverse outcomes.

\section{Methods}

We performed an ambi-directional (predominantly prospective) cohort study of patients with cancer diagnosed with SARS-CoV2 infection between 11th April 2020 and 28th February 2021. The study was approved by the Institutional Ethics Committee, registered with the Clinical Trials Registry of India (CTRI/2020/07/026339) and carried out in accordance with the principles of good clinical research practice.

We included all patients (adult and paediatric) with a proven diagnosis of cancer at any stage of management [under evaluation, on active treatment (curative or palliative intent) or on follow-up (short or long-term)], with SARS CoV2 infection confirmed by a positive RT-PCR test during the study period; those patients with a clinical or radiological suspicion of COVID-19, who tested negative on an RT-PCR test were not included. During the study period, the institutional policy on COVID-19 testing included testing all patients who had symptoms suggestive of COVID and those planned for surgery or other aerosolgenerating procedures.

We collected data from electronic medical records for the following variables: age, sex, co-morbidities needing active treatment, smoking status (ever vs never-smoker), date of diagnosis of cancer, type of cancer (solid vs hematological), intent of management (curative versus palliative), status of management 
(evaluation, active treatment, follow-up), type of management (chemotherapy, radiation therapy, surgery, palliative care, combination or other), date of completion of last systemic anti-cancer treatment (SACT) (defined as chemotherapy, immunotherapy, targeted therapy or a combination of these), date of COVID diagnosis, severity of COVID-19 (classified as per the WHO ordinal scale) ${ }^{13}$ and COVID-19 outcome (dead or alive). The WHO ordinal scale uses the intervention used to treat COVID-19 as a measure of severity, and not the symptoms, and hence would underestimate the severity in situations where care was not escalated due to the terminal nature of the comorbid disease like cancer or an overwhelmed health system. Therefore, we used a composite outcome of severe COVID (WHO grade 6 or more) or death within 30 days from the date of COVID diagnosis as our primary outcome. We used a multivariable logistic regression model to look at association between independent predictors - age, sex, smoking status, presence of comorbidities, type of cancer (solid versus hematological), intent of management (curative versus palliative), duration from last systemic anti-cancer treatment to COVID diagnosis (less than versus more than 30 days) and the primary outcome. We also conducted a similar multivariable analysis to identify risk factors for 30-day mortality. Patients were censored at the last date of follow-up and statistical tests were interpreted at a two-tailed $5 \%$ level of significance.

\section{Results}

We collected data from 1253 patients with a confirmed diagnosis of cancer and SARS CoV2 diagnosed between 11th April 2020 and 28th February 2021. There were 730 male (58\%) and 523 female (42\%) patients; the mean age was 44 (+/-18) years (range 1 to 84 years). The baseline characteristics of the patients are described in Table 1. At a median follow-up of 76 days, 160 patients $(12.8 \%)$ met the composite end-point of severe COVID or death within 30 days of COVID diagnosis. The 30-day all-cause mortality was $10.9 \%$ (138 deaths).

\section{Table-1: Baseline characteristics}




\begin{tabular}{|c|c|c|}
\hline \multirow[t]{3}{*}{ Age in years } & Less than 18 & $\begin{array}{l}161 \\
(12.8)\end{array}$ \\
\hline & 18 to 64 & $\begin{array}{l}933 \\
(74.5)\end{array}$ \\
\hline & 65 and above & $\begin{array}{l}159 \\
(12.7)\end{array}$ \\
\hline \multirow[t]{2}{*}{ Gender } & Male & $\begin{array}{l}730 \\
(58.3)\end{array}$ \\
\hline & Female & $\begin{array}{l}523 \\
(41.7)\end{array}$ \\
\hline \multirow[t]{2}{*}{ Smoking $(n=1227)$} & Yes & $\begin{array}{l}186 \\
(15.2)\end{array}$ \\
\hline & No & $\begin{array}{l}1041 \\
(84.8)\end{array}$ \\
\hline \multirow[t]{2}{*}{ Hypertension $(n=1240)$} & Yes & $\begin{array}{l}209 \\
(16.9)\end{array}$ \\
\hline & No & $\begin{array}{l}1031 \\
(83.1)\end{array}$ \\
\hline \multirow[t]{2}{*}{ Diabetes $(n=1241)$} & Yes & $\begin{array}{l}185 \\
(14.9)\end{array}$ \\
\hline & No & $\begin{array}{l}1056 \\
(85.1)\end{array}$ \\
\hline \multirow[t]{2}{*}{ More than 2 comorbidities $(n=1241)$} & Yes & $\begin{array}{l}148 \\
(11.9)\end{array}$ \\
\hline & No & $\begin{array}{l}1093 \\
(88.1)\end{array}$ \\
\hline \multirow[t]{2}{*}{$\begin{array}{l}\text { Polypharmacy ( } 5 \text { or more medications) } \\
(n=1243)\end{array}$} & Yes & $\begin{array}{l}73 \\
(5.9)\end{array}$ \\
\hline & No & $\begin{array}{l}1170 \\
(94.1)\end{array}$ \\
\hline \multirow[t]{2}{*}{ Cancer diagnosis } & Hematolymphoid & $\begin{array}{l}293 \\
(23.4)\end{array}$ \\
\hline & Solid & $\begin{array}{l}960 \\
(76.6)\end{array}$ \\
\hline \multirow[t]{3}{*}{ Cancer details } & Oral / oropharyngeal & $\begin{array}{l}135 \\
(10.8)\end{array}$ \\
\hline & Breast & $\begin{array}{l}114 \\
(9.1)\end{array}$ \\
\hline & ALL & 107 \\
\hline
\end{tabular}




$\mathrm{NHL}$

Bone

Pancreas

CNS

Prostate 
On active treatment

Chemotherapy

Targeted therapy

Immunotherapy

3

Multimodality

Radiation

Surgery 78

Combination( chemo+targeted therapy) 51

Others (hormonal therapy, steroids, interventional procedures, trans-arterial chemotherapy)

Follow-up

whether on systemic anti-cancer

Yes

COVID

\begin{tabular}{ll}
\hline Chemotherapy & 337 \\
\hline Targeted therapy & 54 \\
\hline Immunotherapy & 2 \\
\hline Combination & 46 \\
\hline No & 814 \\
& $(65.0)$ \\
\hline
\end{tabular}

\section{Data represents actual numbers with percentages in parentheses}

* Other cancers include skin, soft tissue, kidney, larynx, uterus, hypopharynx, nasopharynx, testis, penis, vulva, mediastinum, melanoma, appendix, adrenal.

The severity of COVID was mild (grade 1 to 3 on WHO ordinal scale) in 1014 (81\%) patients, moderate (WHO grade 4 or 5) in 167 (13\%) patients and severe (WHO grade 6 or 7) in 72 (6\%) patients. In patients with limited life expectancy due to advanced cancer, who were considered unsuitable for escalation of therapy, the severity of COVID was graded as per the treatment provided and therefore, may have under- 
estimated the actual severity. All-cause 30-day mortality was 2.4\% (24/1014), 38.3\% (64/167) and 69.4\% $(50 / 72)$ respectively in patients with mild, moderate and severe COVID.

\section{Table - 2: Risk factors for Composite outcome}




\begin{tabular}{|c|c|c|c|c|c|c|}
\hline & & \multirow{2}{*}{$\begin{array}{l}\text { Composite } \\
\text { outcome }\end{array}$} & \multicolumn{2}{|l|}{$\begin{array}{l}\text { Unadjusted } \\
\text { analysis }\end{array}$} & \multicolumn{2}{|c|}{ Adjusted analysis } \\
\hline & & & $\begin{array}{l}\text { OR with } \\
95 \% \mathrm{Cl}\end{array}$ & $\begin{array}{l}P \\
\text { value }\end{array}$ & $\begin{array}{l}\text { OR with } \\
95 \% \mathrm{Cl}\end{array}$ & $\begin{array}{l}P \\
\text { value }\end{array}$ \\
\hline \multirow[t]{3}{*}{ Age (years) } & $\begin{array}{l}\text { Less } \\
\text { than } 18\end{array}$ & $\begin{array}{l}9 / 161 \\
(6 \%)\end{array}$ & reference & & reference & \\
\hline & 18 to 65 & $\begin{array}{l}116 / 933 \\
(13 \%)\end{array}$ & $\begin{array}{l}2.40(1.19 \\
\text { to } 4.83)\end{array}$ & 0.01 & $\begin{array}{l}1.84 \\
(0.86 \\
3.94)\end{array}$ & 0.12 \\
\hline & $\begin{array}{l}\text { More } \\
\text { than } 65\end{array}$ & $\begin{array}{l}35 / 159 \\
(22 \%)\end{array}$ & $\begin{array}{l}4.77(2.21 \\
\text { to } 10.30)\end{array}$ & $<0.01$ & $\begin{array}{l}3.21 \\
(1.36 \text { to } \\
7.56)\end{array}$ & 0.01 \\
\hline \multirow[t]{2}{*}{ Gender } & Female & $\begin{array}{l}69 / 523 \\
(13 \%)\end{array}$ & ref & & ref & \\
\hline & Male & $\begin{array}{l}91 / 730 \\
(13 \%)\end{array}$ & $\begin{array}{l}0.94(0.67 \\
\text { to } 1.31)\end{array}$ & 0.70 & $\begin{array}{l}0.76 \\
(0.51 \text { to } \\
1.14)\end{array}$ & 0.18 \\
\hline \multirow[t]{2}{*}{ Smoking } & No & $\begin{array}{l}120 / 1041 \\
(12 \%)\end{array}$ & ref & & ref & \\
\hline & Yes & $\begin{array}{l}36 / 186 \\
(19 \%)\end{array}$ & $\begin{array}{l}1.84(1.22 \\
\text { to } 2.78)\end{array}$ & 0.004 & $\begin{array}{l}1.78 \\
(1.10 \\
2.91)\end{array}$ & 0.01 \\
\hline \multirow[t]{2}{*}{ More than 2 comorbidities } & No & $\begin{array}{l}125 / 1093 \\
(12 \%)\end{array}$ & ref & & ref & \\
\hline & Yes & $\begin{array}{l}32 / 148 \\
(22 \%)\end{array}$ & $\begin{array}{l}2.14(1.39 \\
\text { to } 3.30)\end{array}$ & 0.01 & $\begin{array}{l}1.66 \\
(1.03 \text { to } \\
2.67)\end{array}$ & 0.04 \\
\hline \multirow[t]{2}{*}{ Cancer type } & Solid & $\begin{array}{l}122 / 960 \\
(13 \%)\end{array}$ & ref & & ref & \\
\hline & $\mathrm{HL}$ & $\begin{array}{l}38 / 293 \\
(13 \%)\end{array}$ & $\begin{array}{l}1.02(0.69 \\
\text { to } 1.51)\end{array}$ & 0.91 & $\begin{array}{l}1.54 \\
(0.96 \text { to } \\
2.48)\end{array}$ & 0.08 \\
\hline \multirow[t]{2}{*}{ Treatment intent } & Curative & $\begin{array}{l}70 / 900 \\
(8 \%)\end{array}$ & ref & & ref & \\
\hline & Palliative & $\begin{array}{l}88 / 344 \\
(26 \%)\end{array}$ & $\begin{array}{l}4.08(2.89 \\
\text { to } 5.75)\end{array}$ & $<0.01$ & $\begin{array}{l}3.57 \\
(2.48 \text { to } \\
5.12)\end{array}$ & $<0.01$ \\
\hline \multirow[t]{2}{*}{$\begin{array}{l}\text { On active SACT within } 30 \\
\text { days prior to COVID diagnosis }\end{array}$} & No & $\begin{array}{l}99 / 812 \\
(12 \%)\end{array}$ & ref & & ref & \\
\hline & Yes & $\begin{array}{l}61 / 439 \\
(14 \%)\end{array}$ & $\begin{array}{l}1.16(0.83 \\
\text { to } 1.64)\end{array}$ & 0.39 & $\begin{array}{l}1.09 \\
(0.72 \text { to } \\
1.63)\end{array}$ & 0.70 \\
\hline
\end{tabular}


In a separate multivariable logistic regression analysis with 30-day mortality as the primary outcome, we identified advanced age (OR 4.30; $95 \% \mathrm{Cl} 1.59$ to 11.64) and palliative intent of treatment (OR 4.20; $95 \%$ $\mathrm{Cl} 2.84$ to 6.20 ) as independent risk factors. Among patients treated with palliative intent, those who received SACT within 30 days prior to COVID, 25\% (36/145) had an event, compared to $26 \%(52 / 199)$ in those who did not. In patients older than 65 years who received SACT within 30 days prior to COVID, $10 / 34$ (29\%) experienced the composite end-point compared to $20 \%(25 / 125)$ in those who had not received SACT.

\section{Discussion}

In this study of patients with cancer who developed COVID-19, we identified advanced age, history of smoking, palliative intent of treatment and presence of more than 2 comorbidities to be risk factors for severe COVID or death within 30 days. Advanced age and palliative intent of treatment remained independently associated with 30-day mortality. Recent systemic anti-cancer therapy, gender and cancer type were not significantly associated with adverse outcomes.

Since the onset of the COVID-19 pandemic, there have been concerns about the outcomes of COVID-19 in patients with cancer. A meta-analysis of 26 studies including 23736 patients with cancer found a pooled all-cause in-hospital mortality rate of $19 \%$, with nearly three times higher odds of dying than those without cancer. ${ }^{4}$ Early data from China suggested that patients with cancer and COVID had a considerably poorer survival than the general population with mortality estimates between 20 and $29 \% .{ }^{14-17}$ These studies were limited by their small sample sizes and largely retrospective nature. Subsequent studies from other parts of the world reported short-term mortality rates between $10 \%$ and $29 \%$ in patients with cancer and COVID, with the UK and some countries in Europe reporting much higher fatality rates than other parts of the world. ${ }^{5-7,18-21}$ The results from our study are at the lower end of the published range of fatality data on COVID-19 outcomes in patients with cancer.

The dissimilarity in results between these quoted studies need to be interpreted keeping in mind that they were done at various time periods corresponding to different phases of the pandemic. Studies at the beginning of the pandemic typically reported higher case fatality rates as very little was known about the disease and its management. Also, differences in testing strategies between countries may imply that in some studies, patients with cancer who were symptomatic with mild disease and potentially favourable outcomes may not have been identified as opposed to those with moderate and severe disease, resulting in higher estimated fatality rates. Another factor which needs to be considered is the indication for hospital admission. Our study and other studies from India ${ }^{10,11}$ reported only 4 to $11 \%$ of severe COVID cases among those admitted, compared to 20 to $45 \%$ in other studies. ${ }^{5,6,18,21}$ Inadequate facilities for home isolation, possibly led to a number of hospital admissions for social reasons in patients with mild disease, leading to a reduction in the proportion of those with severe disease and a decrease in hospital mortality. In addition, the outcomes of patients with cancer and COVID-19 need to be compared to the outcomes in the general population for that same country. Countries such as Italy and the UK have 
reported population case fatality rates of 3 to $5 \%$ compared to $1.1 \%$ in India ${ }^{1}$ These differences could be partly related to population characteristics, with developed countries having a high proportion of elderly individuals with comorbidities. ${ }^{22}$ For example, many African countries where the life expectancy is low and the population is predominantly young have reported very low COVID fatality rates. ${ }^{23}$ Other associated factors include time trends in the spread of the pandemic, capacity and strategy for testing, and the accuracy of reporting deaths. ${ }^{22}$ The low COVID fatality rate in India could also be because of the decreased severity of infection, possibly due to cross-immunity from exposure to other coronaviruses which are endemic in the population. ${ }^{9}$

The focus of research on COVID outcomes in patients with cancer has been to identify prognostic factors to aid risk stratification and early recognition of patients likely to have adverse outcomes. In keeping with the published literature, we found that advanced age was an independent risk factor for poor outcomes after COVID, and within this group, elderly patients who had received recent systemic anti-cancer therapy had worse outcomes than those who did not. ${ }^{6,17,18}$ Similar to other studies, we found that the presence of comorbidities and smoking adversely affected COVID severity and outcomes. ${ }^{6,21}$ Our study also showed no impact of gender, type of cancer or recent SACT on COVID-19 outcomes. These findings strongly support the continuation of cancer care in most patients during future surges of the pandemic.

Our results showed that treatment with palliative intent was a significant adverse prognostic factor for COVID outcomes, regardless of whether active anti-cancer treatment had been recently administered. This can be attributed to the debilitation caused by the cancer itself, compounded by the effects of COVID. Paradoxically, Di Cosimo showed that patients with metastatic disease receiving anti-cancer therapy had better COVID outcomes than their untreated counterparts; ${ }^{24}$ however, this is possibly confounded by selection bias as patients with better general health and performance status are more likely to receive active treatment. Our study suggests that the treatment of patients with advanced, metastatic cancers should be guided by the magnitude of benefit based on the de-novo or progressive nature of cancer, expected toxicities with treatment and potential risks of COVID-19 related complications. This highlights that emphasis should be given to shared-decision making in this scenario. This is particularly true when healthcare systems are overwhelmed by COVID-19, and the resources diverted to palliative chemotherapy would be at the expense of delivery of care to those with other disease conditions, including patients with cancer who are on treatment with curative intent.

Initial data from China suggested that recent SACT was a risk factor for COVID severity and mortality. ${ }^{14}$ Based on this, several international organizations issued guidelines for the management of cancer during the pandemic, which included risk minimization by care prioritization, de-intensification of therapies, and postponing therapy where possible. ${ }^{25}$ Subsequent studies from other parts of the world have shown differing results - while some studies showed that recent SACT, especially chemotherapy and immunotherapy were risk factors for severity of infection or mortality, other data showed no evidence of an association. ${ }^{5,6,7,18,19}$ A systematic review found that chemotherapy within 30 days prior to diagnosis of COVID increased the risk of death but not of severe COVID while other therapies (including radiation 
and immunotherapy) had no such effect. ${ }^{26}$ While this may be explained on the basis of the intense immunosuppression caused by chemotherapy, it needs to be interpreted cautiously. First, many studies have not been able to capture reliable data on the nature and timing of systemic therapy in relation to COVID and the available data is quite limited. Second, studies may have grouped all forms of anti-cancer therapy which would dilute the effect of individual treatments. Third, changes in practice during the pandemic may have resulted in only fitter patients receiving intense chemotherapy, thus confounding the results.

Our study has several strengths. First, to the best of our knowledge, it is one of the largest single-centre studies examining the outcomes of COVID-19 in patients with cancer, and is possibly the most robust prospective data available from this part of the world. Second, this was a pragmatic study which included all patients regardless of age, cancer type or severity of COVID-19. Finally, being a referral centre in the state for patients with cancer who developed COVID-19, it is likely to be fairly representative of the realworld situation. One possible limitation is that a small proportion of patients who were relatively less symptomatic, but did not have facilities for home isolation were admitted in hospital for social rather than medical reasons, thus potentially skewing the severity scoring of the illness; however, these numbers were few; moreover, since we used categories of disease (mild, moderate and severe) rather than actual scores, this is unlikely to have influenced the results.

The results of our study have important policy-level implications. We have demonstrated that in our setting, most patients with cancer who developed COVID-19 had mild disease and favourable outcomes. Considering that India has a huge burden of COVID cases and has had multiple surges in the number of active infections, this finding is important to assuage fear in patients and treatment-providers. With increasing realization of the adverse outcomes that are likely by deferring active cancer treatment, our results support the continuation of cancer care even during pandemics. Treatment of cancer during the pandemic has been severely hampered due to multiple reasons: inability of patients to access care due to fear of contracting COVID or travel restrictions, reduction in existing cancer care facilities either because of conversion to COVID care centres or due to staffing issues (illness, quarantine or travel restrictions) and recommendations to downscale or delay cancer therapies. A study across 41 cancer centres in India found substantial reductions in volumes of patients treated at these centres during the peak of the pandemic. ${ }^{27}$ Even in the pre-pandemic period, several low and middle income countries faced challenges with cancer care related to lack of access, delayed stage presentation and poor outcomes. ${ }^{28}$ In such settings, further reductions in cancer care are likely to have disastrous consequences. Many of these countries are now seeing new waves of COVID infections and the findings of this study reinforce that cancer care should not be deprioritized even during a pandemic.

\section{Declarations}

We declare that the authors have no competing interests as defined by Nature Research, or other interests that might be perceived to influence the results and/or discussion reported in this paper. 


\section{References}

1. WHO Coronavirus (COVID 19) dashboard. Available at https://covid19.who.int/ Last accessed on 17th October 2021

2. Booth A, Reed AB, Ponzo S, Yassaee A, Aral M, Plans D, et al. Population risk factors for severe disease and mortality in COVID-19: A global systematic review and meta-analysis. PLoS One. 2021;16:e0247461

3. Flook M, Jackson C, Vasileiou E, Simpson CR, Muckian MD, Agrawal U,. Informing the public health response to COVID-19: a systematic review of risk factors for disease, severity, and mortality. BMC Infect Dis. 2021;21:342.

4. Venkatesulu BP, Chandrasekar VT, Girdhar P, Advani P, Sharma A, Elumalai T, et al. A Systematic Review and Meta-Analysis of Cancer Patients Affected by a Novel Coronavirus. JNCI Cancer Spectr. 2021;5:pkaa102.

5. Robilotti EV, Babady NE, Mead PA, Rolling T, Perez-Johnston R, Bernardes M, et al. Determinants of COVID-19 disease severity in patients with cancer. Nat Med. 2020;26:1218-1223.

6. Kuderer NM, Choueiri TK, Shah DP, Shyr Y, Rubinstein SM, Rivera DR, et al; COVID-19 and Cancer Consortium. Clinical impact of COVID-19 on patients with cancer (CCC19): a cohort study. Lancet. 2020;395:1907-1918.

7. Johannesen TB, Smeland S, Aaserud S, Buanes EA, Skog A, Ursin G, Helland A. COVID-19 in Cancer Patients, Risk Factors for Disease and Adverse Outcome, a Population-Based Study From Norway. Front Oncol. 2021;11:652535.

8. Anand A. Three New Estimates of India's All-Cause Excess Mortality during the COVID-19 Pandemic. https://cgdev.org/publication/three-new-estimates-indias-all-cause-excess-mortality-during-covid-19pandemic.

9. Chakrabarti SS, Kaur U, Banerjee A, Ganguly U, Banerjee T, Saha S, et al. COVID-19 in India: Are Biological and Environmental Factors Helping to Stem the Incidence and Severity? Aging Dis. 2020;11:480-488.

10. Ramaswamy A, Nayak L, Roy Moulik N, Sengar M, Chinnaswamy G, Jobanputra K, et al. COVID-19 in cancer patients on active systemic therapy - Outcomes from LMIC scenario with an emphasis on need for active treatment. Cancer Med. 2020;9:8747-8753.

11. Mehta A, Vasudevan S, Parkash A, Sharma A, Vashist T, Krishna V. COVID-19 mortality in cancer patients: a report from a tertiary cancer centre in India. PeerJ. 2021;9:e10599.

12. Radhakrishnan V, Ovett J, Rajendran A, Kolluru S, Pai V, Gnanaguru V, et al. COVID19 in children with cancer in low- and middle-income countries: Experience from a cancer center in Chennai, India. Pediatr Hematol Oncol. 2021;38:161-167.

13. WHO R\&D Blueprint novel Coronavirus COVID-19 Therapeutic Trial Synopsis. World Health Organization 2020. Available at https://www.who.int/blueprint/priority-diseases/key-action/COVID- 
19_Treatment_Trial_Design_Master_Protocol_synopsis_Final_18022020.pdf. Accessed on Oct 15, 2021

14. Zhang L, Zhu F, Xie L, Wang C, Wang J, Chen R, et al. Clinical characteristics of COVID-19-infected cancer patients: a retrospective case study in three hospitals within Wuhan, China. Ann Oncol. 2020;31:894-901.

15. Tian J, Yuan X, Xiao J, Zhong Q, Yang C, Liu B, et al. Clinical characteristics and risk factors associated with COVID-19 disease severity in patients with cancer in Wuhan, China: a multicentre, retrospective, cohort study. Lancet Oncol. 2020;21:893-903.

16. Yang F, Shi S, Zhu J, Shi J, Dai K, Chen X. Clinical characteristics and outcomes of cancer patients with COVID-19. J Med Virol. 2020;92:2067-2073.

17. Meng Y, Lu W, Guo E, Liu J, Yang B, Wu P, et al. Cancer history is an independent risk factor for mortality in hospitalized COVID-19 patients: a propensity score-matched analysis. J Hematol Oncol. 2020;13:75.

18. Lee LY, Cazier JB, Angelis V, Arnold R, Bisht V, Campton NA, et al; UK Coronavirus Monitoring Project Team, Kerr R, Middleton G. COVID-19 mortality in patients with cancer on chemotherapy or other anticancer treatments: a prospective cohort study. Lancet. 2020;395:1919-1926.

19. Pinato DJ, Lee AJX, Biello F, Seguí E, Aguilar-Company J, Carbó A, et al. Presenting Features and Early Mortality from SARS-CoV-2 Infection in Cancer Patients during the Initial Stage of the COVID-19 Pandemic in Europe. Cancers (Basel). 2020;12:1841.

20. Russell B, Moss C, Papa S, Irshad S, Ross P, Spicer J, et al. Factors Affecting COVID-19 Outcomes in Cancer Patients: A First Report From Guy's Cancer Center in London. Front Oncol. 2020;10:1279.

21. Lièvre A, Turpin A, Ray-Coquard I, Le Malicot K, Thariat J, Ahle G, et al; GCO-002 CACOVID-19 collaborators/investigators. Risk factors for Coronavirus Disease 2019 (COVID-19) severity and mortality among solid cancer patients and impact of the disease on anticancer treatment: A French nationwide cohort study (GCO-002 CACOVID-19). Eur J Cancer. 2020;141:62-81.

22. Sorci G, Faivre B, Morand S. Explaining among-country variation in COVID-19 case fatality rate. Sci Rep. 2020;10:18909.

23. Lawal Y. Africa's low COVID-19 mortality rate: A paradox? Int J Infect Dis. 2021;102:118-122.

24. Di Cosimo S, Porcu L, Malfettone A, Cortés J, Miceli R. Commentary: SARS-CoV-2 Transmission in Patients With Cancer at a Tertiary Care Hospital in Wuhan, China. Front Oncol. 2020;10:1223.

25. Hanna TP, Evans GA, Booth CM. Cancer, COVID-19 and the precautionary principle: prioritizing treatment during a global pandemic. Nat Rev Clin Oncol. 2020;17:268-270.

26. Yekedüz E, Utkan G, Ürün Y. A systematic review and meta-analysis: the effect of active cancer treatment on severity of COVID-19. Eur J Cancer. 2020;141:92-104.

27. Ranganathan P, Sengar M, Chinnaswamy G, Agrawal G, Arumugham R, Bhatt R, et al; National Cancer Grid of India. Impact of COVID-19 on cancer care in India: a cohort study. Lancet Oncol. 2021;22:970-976. 
28. Pramesh CS, Badwe RA, Borthakur BB, Chandra M, Raj EH, Kannan T, et al. Delivery of affordable and equitable cancer care in India. Lancet Oncol. 2014;15:e223-33. 